\title{
AN IMAGE EDGE DETECTION METHOD BASED ON MATHEMATICAL MORPHOLOGY
}

\author{
Ji Xiaoping ${ }^{1}$, Zhang Haibin ${ }^{2}$, Zhang Dazhi ${ }^{1}$, Wu Boying ${ }^{1}$
}

\begin{abstract}
We present two kinds of improved morphological edge detectors according to: the characteristics of the classical morphological operators and the disadvantages of the single structural elements selected. The structure of the first improved operator considers multi-structural elements and omni-directional structural elements, along with the characteristics of dilation, erosion, opening and closing. The second improved operator uses a different structural element based on the first improved operator, which is characterized by multi-structure, multi-scale and multi-direction. The final experiment's results show that, comparing with the classical morphological operators and traditional edge detectors, the edge detection effects and the denoising ability of improved operators are superior, and they are extremely practical, and can be widely used in subsequent image processing.
\end{abstract}

Keywords Mathematical morphology · Edge detection . Structural elements . Denoising ability

\author{
${ }^{1}$ Jixiaoping \\ Department of Mathematics Harbin Institute of Technology Harbin, China \\ xiaopingji@hit.edu.cn \\ ${ }^{2}$ Zhanghaibin \\ HeiLongJiang electic power co., LTD \\ zhanghaibin@hl.sgcc.com.cn \\ ${ }^{1}$ Zhangdazhi $(\square)$ \\ Department of Mathematics Harbin Institute of Technology, No. 92, Xidazhi Street, \\ Harbin, 150001, PR China \\ zhangdazhi@ hit.edu.cn \\ ${ }^{1}$ Wuboying \\ Department of Mathematics Harbin Institute of Technology Harbin, China \\ mathwby@hit.edu.cn
}




\section{Introduction}

Image edge is the most essential feature of an image, and image edge detection is an important part of the image pre-processing and analysis, and has a wide range of theoretical and practical significance. The image edge refers to the place where grayscale changes is more obvious and severe (also known as grayscale mutation), namely grayscale is not continuous to obtain the result, essentially means the end of an area and the beginning of another area. Traditional edge detection method uses first-order gradient or zero-crossing value of second derivative to detect edge, including the Roberts edge detector, Sobel edge detector, Prewitt edge detector, Laplacian edge detector, LOG edge detector, Canny edge detector and so on. These methods have many problems, especially in resolving the conflict between denoising ability and detection accuracy, so the experimental results are not satisfactory. While mathematics morphology method may detect accurate edge at the same time restrain the noise well [1]. Mathematical morphology for image processing and analysis methods are nonlinear, whose theoretical foundation is set theory, First transforms the image into the set with a correct way, then carries on the basic operation with the way of shift, intersection, union and complement[2]. The basic idea of mathematical morphology is to carry on the analysis and the recognition for the image. We adopt a certain modal structuring elements to measure and extract the corresponding shape in the image. Edge detection algorithm based on mathematical morphology is better than edge detection algorithm based on differential; it can overcome the effect of noise by making the scale of the morphological structuring elements change during the edge detection.

Using mathematical morphological method for edge detection, we can choose certain structuring elements to dilate and erode the image. The original image subtracting the eroded image and the dilated image subtracting the original image can obtain the image edge, this can make the image edge information maintain well. Using morphological operation by selecting the appropriate structuring elements, we can filter out the noise, simultaneously enable the original image information be retained well. This is greatly superior to the traditional edge detection methods, because it is able to extract more smooth edges, and is not sensitive to noise, which can meet the real-time requirements, and be easily implemented in hardware.

\section{The Basic Theory of Mathematical Morphology in Image Processing}

Mathematical morphology is a new method for processing and identifying the image on the basis of set theory, the basic principle of whose is that the image is regarded as a point set and carrying on the set operation[3, 4]. This forms a variety of morphological processing algorithm, namely that using structuring elements to 
carry on shift, intersection, union and complement operations. The structuring elements are also regarded as point set, that move constantly in the image to achieve the purpose of image analysis and processing at the same time. The different point sets constitute the structuring elements with different characteristics, and that can detect characteristics of the image with different sides, so we may regard structuring elements as a mean of image processing.

Definition 2.1 [5] Let the input image be $f(x, y)$, and structuring element be $b(x, y)$, then

(i) The grayscale erosion of $f(x, y)$ by $b(x, y)$, denoted $f \Theta b$, is defined as:

$$
\begin{aligned}
& (f \Theta b)(s)=\min \\
& \left\{f(s+x, t+y)-b(x) \mid(s+x) \in D_{f} ; x \in D_{b}\right\}
\end{aligned}
$$

(ii) The grayscale dilation of $f(x, y)$ by $b(x, y)$, denoted $f \oplus b$, is defined as:

$$
\begin{aligned}
& (f \oplus b)(s)=\max \\
& \left\{f(s-x, t-y)+b(x) \mid(s-x) \in D_{f} ; x \in D_{b}\right\}
\end{aligned}
$$

(iii) The opening of $f(x, y)$ by $b(x, y)$, denoted $f \circ b$, is defined as: $f \circ b=(f \Theta b) \oplus b$

(iv) The closing of $f(x, y)$ by $b(x, y)$, denoted $f \bullet b$, is defined as: $f \bullet b=(f \oplus b) \Theta b$

Where $D_{f}$ and $D_{b}$ are respectively the domain of $f(x, y)$ and $b(x, y)$, simultaneously $(s+x, t+y)$ and $(s-x, t-y)$ are in the domain of $f(x, y)$.

Erosion can weaken the smaller and brighter parts, comparing with structuring elements in the grayscale image, so that the grayscale image will be darker than the original image; Dilation is just the opposite, for it can eliminate the smaller and darker parts comparing with structuring elements, so that the grayscale image will be brighter than the original image. Opening can eliminate the smaller luminescent spots comparing with structuring elements in the image, and make characteristics of all gray and larger bright area be maintained basically invariable (The reason is that first erosion can eliminate the smaller bright details and weaken the brightness of the image, while later dilation can restore basically bright detail parts of the image and already removed part is not to be introduced); Closing can eliminate the smaller dim spots comparing with structuring elements in the image, and make characteristics of all gray and larger dark area be maintained basically invariable(The reason is similar in opening, that first dilation can eliminate the smaller dark details and enhance the brightness of the image, while later erosion can recover dark information of the image and already removed part is not to be introduced). Combination of opening and closing can 
achieve a morphological smoothing, and constitute the morphological filter. It can be constructed as follows several basic morphological gradients[6], including dilation type denoted $\mathrm{Grad}_{1}$, erosion type denoted $\mathrm{Grad}_{2}$, dilation-erosion type denoted $\mathrm{Grad}_{3}$, opening type denoted $\mathrm{Grad}_{4}$, closing type denoted $\mathrm{Grad}_{5}$ and opening-closing type denoted $\mathrm{Grad}_{6}$, are defined as follows: $\operatorname{Grad}_{1}=f \oplus B-f$, $\operatorname{Grad}_{2}=f-f \Theta B, \operatorname{Grad}_{3}=f \oplus B-f \Theta B, \operatorname{Grad}_{4}=f-f \circ B, \operatorname{Grad}_{5}=f \bullet B-f$, $\operatorname{Grad}_{6}=f \bullet B-f \circ B$.

The above edge detectors are all nonlinear differential operator, and the edge detection results Relate to the structuring elements. In the practical application process, selecting the different structuring elements can obtain the different edge detection results, and using the different operators can detect the different edge effects. The dilation type operator extract the outer edge of the image, and the erosion type operator extract the inner edge of the image, and the dilation-erosion type operator extract the image edge which is in the actual European borders. This kind of edge detector can obtain the relatively accurate results, which can ensure the connectivity of the original image; however, the above operators are sensitive to noise. Erosion and opening can restrain the positive noise, while dilation and closing can restrain the negative noise, thus Yang Shubin proposes denoising dilation-erosion type operator, denoted $\mathrm{Grad}_{7}$, is defined as: $\mathrm{Grad}_{7}=(f \circ B) \oplus B-(f \bullet B) \Theta B$

Classical morphological operators are susceptible to noise, and the denoising ability of denoising dilation-erosion type operator is obviously stronger, but they only adopt the edge detector of a single structuring element, with only limited denoising ability, and using this single edge detector to detect the edge whose geometrical shape is rich, that is very difficult, so the detection effects are not very ideal.

\section{Improved Morphological Edge Detection}

\section{A. The first improved operator}

We know that the multi-structure elements can detect types of edges, and the omni-directional structuring element can detect the image edge in all directions.

In order to make up for the lack of single structuring elements for edge detection, we propose an improved morphological edge detector, which is considered from the two angles about multi-structure and omni-direction, is defined as follows operator.

Definition 3.1 let the input image be $f(x, y), B_{1}$ and $B_{2}$ be the different structuring elements, then 
(i) Morphological edge detector for salt and pepper noise image, denoted $E(f)$, is defined as:

$$
E(f)=\left[\left(f \bullet B_{1} \circ B_{1}\right) \bullet B_{1}\right] \oplus B_{2}-\left[\left(f \bullet B_{1}\right) \circ B_{1}\right] \Theta B_{2}
$$

(ii) Morphological edge detector for Gaussian noise image, denoted $G(f)$, is defined as:

$$
G(f)=\left[\left(f \circ B_{1} \bullet B_{1}\right) \circ B_{1}\right] \oplus B_{2}-\left[\left(f \bullet B_{1}\right) \circ B_{1}\right] \Theta B_{2}
$$

Where $B_{1}$ is a $3 \times 3$ cruciform structuring element, that is

$$
B_{1}=\left[\begin{array}{lll}
0 & 1 & 0 \\
1 & 1 & 1 \\
0 & 1 & 0
\end{array}\right]
$$

First we do the repeated open-closing filter of the original image by $B_{1}$ to smooth image and eliminate the noise; Then we adopt the $3 \times 3$ quadrate structuring element denoted $B_{2}$ to detect edge, where $B_{2}$ is broken down into four different directional structuring elements denoted $B_{2 i}(i=1,2,3,4)$, that is $B_{2}=\bigcup_{i=1}^{4} B_{2 i}$, and

$$
\begin{gathered}
B_{21}=\left[\begin{array}{lll}
0 & 0 & 0 \\
1 & 1 & 1 \\
0 & 0 & 0
\end{array}\right] B_{22}=\left[\begin{array}{lll}
0 & 0 & 1 \\
0 & 1 & 0 \\
1 & 0 & 0
\end{array}\right] \quad B_{23}=\left[\begin{array}{lll}
0 & 1 & 0 \\
0 & 1 & 0 \\
0 & 1 & 0
\end{array}\right] \quad B_{24}=\left[\begin{array}{lll}
1 & 0 & 0 \\
0 & 1 & 0 \\
0 & 0 & 1
\end{array}\right] \\
B_{2}=\left[\begin{array}{lll}
1 & 1 & 1 \\
1 & 1 & 1 \\
1 & 1 & 1
\end{array}\right]
\end{gathered}
$$

These four structuring elements basically cover all directions of $B_{2}$, that makes the edge in all directions be detected by this operator. This operator is suitable for image with detection of complex edges, and can get complete edge.

\section{B. The second improved operator}

We know that the small scale structuring elements which have the poor denoising ability, but it can maintain the edge details well; While large-scale structuring elements which have the better denoising ability, but it make some of the edge details be lost at the same time. Therefore, we consider two structuring elements with different scales filtering alternately, that can not only restrain the noise effectively, but also can maintain the edge details well.

The following improved morphological edge detection operator is a combination of three factors about multi-structure, multi-scale and omnidirectional is defined as follows. 
Definition 3.2 Let the input image be $f(x, y), B_{1}, B_{3}$ and $B_{4}$ be the different structuring elements, then

(i) Morphological edge detector for salt and pepper noise image, denoted $E(f)$, is defined as:

$$
E(f)=\left[\left(f \bullet B_{1} \circ B_{1}\right) \bullet B_{3}\right] \oplus B_{4}-\left[\left(f \bullet B_{1}\right) \circ B_{3}\right] \Theta B_{4}
$$

(ii) Morphological edge detector for Gaussian noise image, denoted $G(f)$, is defined as:

$$
G(f)=\left[\left(f \circ B_{1} \bullet B_{1}\right) \circ B_{3}\right] \oplus B_{4}-\left[\left(f \bullet B_{1}\right) \circ B_{3}\right] \Theta B_{4}
$$

Where the value of $B_{1}$ is with reference to the first improved operator, $B_{3}$ is a $5 \times 5$ diamond-shaped structuring element, that is

$$
B_{3}=\left[\begin{array}{lllll}
0 & 0 & 1 & 0 & 0 \\
0 & 1 & 1 & 1 & 0 \\
1 & 1 & 1 & 1 & 1 \\
0 & 1 & 1 & 1 & 0 \\
0 & 0 & 1 & 0 & 0
\end{array}\right]
$$

First we do the alternate open-closing filter of the original image by $B_{1}$ and $B_{3}$ to smooth image and eliminate the noise; Then we adopt the $5 \times 5$ quadrate structuring element denoted $B_{4}$ to detect edge, where $B_{4}$ is broken down into eight different directional structuring elements denoted $B_{4 i}(i=1,2, \cdots, 8)$, that is $B_{4}=\bigcup_{i=1}^{8} B_{4 i}$, and

$$
\begin{aligned}
& B_{41}=\left[\begin{array}{lllll}
0 & 0 & 0 & 0 & 0 \\
0 & 0 & 0 & 0 & 0 \\
1 & 1 & 1 & 1 & 1 \\
0 & 0 & 0 & 0 & 0 \\
0 & 0 & 0 & 0 & 0
\end{array}\right] \quad B_{42}=\left[\begin{array}{lllll}
0 & 0 & 0 & 0 & 0 \\
0 & 0 & 0 & 0 & 1 \\
0 & 0 & 1 & 0 & 0 \\
1 & 0 & 0 & 0 & 0 \\
0 & 0 & 0 & 0 & 0
\end{array}\right] \quad B_{43}=\left[\begin{array}{lllll}
0 & 0 & 0 & 0 & 1 \\
0 & 0 & 0 & 1 & 0 \\
0 & 0 & 1 & 0 & 0 \\
0 & 1 & 0 & 0 & 0 \\
1 & 0 & 0 & 0 & 0
\end{array}\right] \\
& B_{44}=\left[\begin{array}{lllll}
0 & 0 & 0 & 1 & 0 \\
0 & 0 & 0 & 0 & 0 \\
0 & 0 & 1 & 0 & 0 \\
0 & 0 & 0 & 0 & 0 \\
0 & 1 & 0 & 0 & 0
\end{array}\right] \quad B_{45}=\left[\begin{array}{lllll}
0 & 0 & 1 & 0 & 0 \\
0 & 0 & 1 & 0 & 0 \\
0 & 0 & 1 & 0 & 0 \\
0 & 0 & 1 & 0 & 0 \\
0 & 0 & 1 & 0 & 0
\end{array}\right] \quad B_{46}=\left[\begin{array}{lllll}
0 & 1 & 0 & 0 & 0 \\
0 & 0 & 0 & 0 & 0 \\
0 & 0 & 1 & 0 & 0 \\
0 & 0 & 0 & 0 & 0 \\
0 & 0 & 0 & 1 & 0
\end{array}\right] \\
& B_{47}=\left[\begin{array}{lllll}
1 & 0 & 0 & 0 & 0 \\
0 & 1 & 0 & 0 & 0 \\
0 & 0 & 1 & 0 & 0 \\
0 & 0 & 0 & 1 & 0 \\
0 & 0 & 0 & 0 & 1
\end{array}\right] \quad B_{48}=\left[\begin{array}{lllll}
0 & 0 & 0 & 0 & 0 \\
1 & 0 & 0 & 0 & 0 \\
0 & 0 & 1 & 0 & 0 \\
0 & 0 & 0 & 0 & 1 \\
0 & 0 & 0 & 0 & 0
\end{array}\right] \quad B_{4}=\left[\begin{array}{lllll}
1 & 1 & 1 & 1 & 1 \\
1 & 1 & 1 & 1 & 1 \\
1 & 1 & 1 & 1 & 1 \\
1 & 1 & 1 & 1 & 1 \\
1 & 1 & 1 & 1 & 1
\end{array}\right]
\end{aligned}
$$


These eight structuring elements basically cover all directions of $B_{4}$, thus we get close to the complete edges.

\section{Algorithm and Experimental Results Analysis}

\section{A. The algorithm of improved operators}

The algorithm steps of the second improved operator are as follows (which the algorithm steps of the first improved operator are similar to):

(i) Do the alternate open-closing filter of the original image by $B_{1}$ and $B_{3}$ to smooth image;

(ii) Carry on the edge detection with eight different directional structuring elements $B_{4 i}(i=1,2, \cdots, 8)$ separately to obtain:

$$
\begin{gathered}
E_{i}(f)=\left[\left(f \bullet B_{1} \circ B_{1}\right) \bullet B_{3}\right] \oplus B_{4 i}-\left[\left(f \bullet B_{1}\right) \circ B_{3}\right] \Theta B \quad \text { or } \\
G_{i}(f)=\left[\left(f \circ B_{1} \bullet B_{1}\right) \circ B_{3}\right] \oplus B_{4 i}-\left[\left(f \bullet B_{1}\right) \circ B_{3}\right] \Theta B_{4 i} i=1,2, \cdots, 8
\end{gathered}
$$

The asked image edges are the weighted sum of each directional edge, that is

$$
E(f)=\sum_{i=1}^{8} \omega_{i} E_{i} \quad \text { or } \quad G(f)=\sum_{i=1}^{8} \omega_{i} G_{i}
$$

Where $\omega_{i}$ is weight coefficient and $\omega_{i}=1 / 8\left(\omega_{i}=1 / 4\right.$ in the first improved operator), because the image has basically no noise after the repeated openclosing filter, such that the edge effects in each direction are consistent.

(iii) Do the post-processing for the edge, namely the detected edge is linearization first and refinement later to get the final results.

\section{B.Experimental simulation and results analysis}

1) The experimental results of edge detection for salt and pepper noise image

Figure 1 and Figure 2 are the effect figures of edge detection, comparing with Canny edge detector which is the best in traditional algorithm and morphology operator in literature[7], where (a) is the image respectively joined in the salt and pepper noise with intensity of 0.01 and 0.03 , and (b) to (e) are the results of every edge detection operator. 


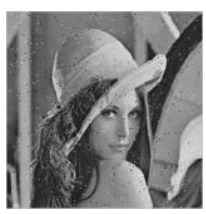

(a)

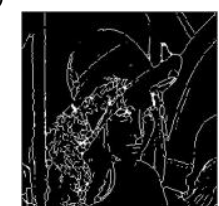

(d)

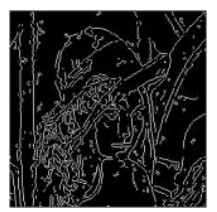

(b)

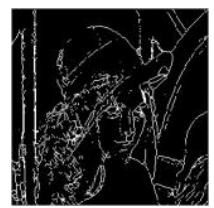

(c)

Fig. 4.1 The effect figures of edge detection

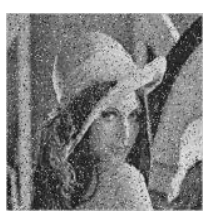

(a)

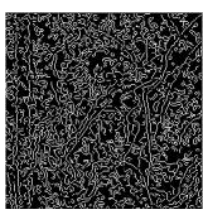

(b)

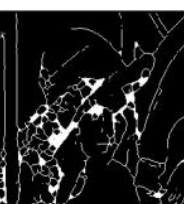

(e)
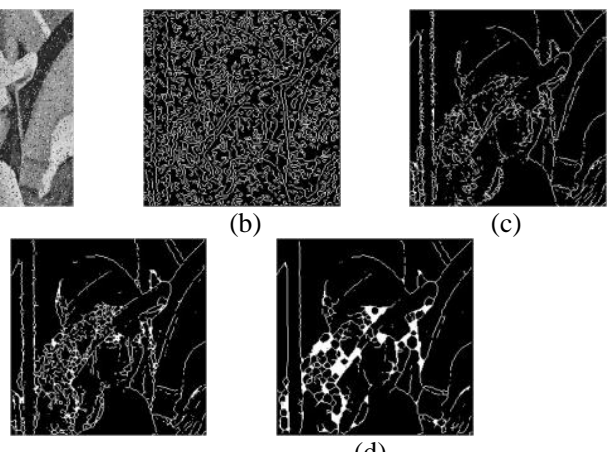

(c)

(d)

(e)

Fig. 4.2 The effect figures of edge detection

Figure 4.1 and Figure 4.2 show that, comparing with three morphology operators(including the denoising dilation-erosion type operator be introduced by literature, the first improved operator and the second improved operator), Canny edge detector is significantly affected by noise seriously, especially after the noise increases, it is not able to distinguish the edge; comparing with the morphology operator in literature, the first improved operator maintains the edge details well, a stronger denoising ability and a better edge smoothness, meanwhile increasing the noise has little effect on the results of edge detection; The second improved operator gets the edge be smoother, the outline be clearer, and denoising ability be stronger, especially increasing the noise does not affect the results of edge detection, but in the edge-intensive place(such as hair and eyes, etc.) the result is some details of the edge to stick together, so the second improved operator is more suitable for the edge detection, that the requirement of the external edge is high and the requirement of the internal edge details is low. 


\section{2) The experimental results of edge detection for Gaussian noise image}

Figure 3 and Figure 4 are the effect figures of edge detection, where (a) is the image respectively joined in the Gaussian noise with mean 0 , variance 0.005 and mean 0 , variance 0.01 , and (b) to (e) are the results of every edge detection operator.

Figure 3 and Figure 4 show that, comparing with three morphology operators, the traditional Canny edge detector is more obvious affected by noise, presents some pseudo- edges; Comparing with the morphology operator in literature, the first improved operator maintains the edge details well, a stronger denoising ability, a better edge smoothness and continuity, meanwhile increasing the noise has little effect on the results of edge detection; Comparing other operators, the second improved operator gets the edge be more smooth and continuous, clear and complete, especially increasing the noise may not affect the results of edge detection, but only some of the edge details are easy as the noise be filtered out.

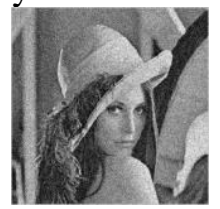

(a)

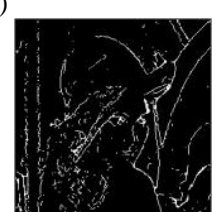

(d)

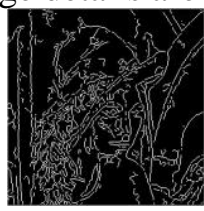

(b)

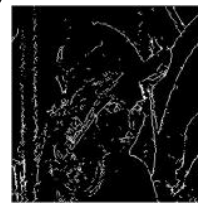

(c)

Fig. 4.3 The effect figures of edge detection

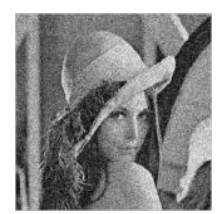

(a)

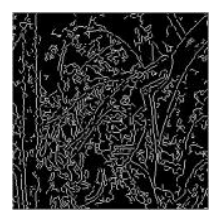

(b)

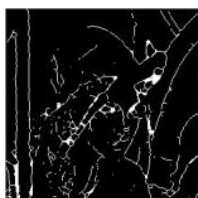

(e)

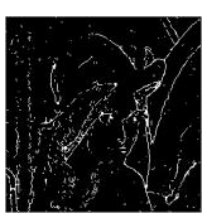

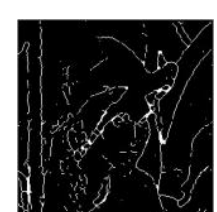

(d)

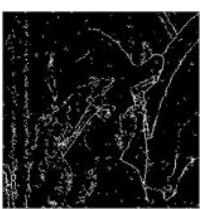

(c)

Fig. 4.4 The effect figures of edge detection 


\section{Conclusions}

This paper is mainly to study and improve the morphological edge detector, propose two new morphological edge detectors. It can be seen from the experiment, the two operators take into account two factors that edge detection accuracy and noise immunity, the edges obtained have characteristics of rich details, high smoothness, strong continuity and good denoising ability, the examination effect is very ideal, and has a strong feasibility and practicality. The two edge detectors provide a great help for the subsequent processing of the image.

\section{Acknowledgment}

This work is supported by the Fundamental Research Funds for the Central Universities (Grant No. HIT. NSRIF. 2012065), the National Science Foundation of China (Grant no. 11271100), the Aerospace Supported Fund, China, under Contract no. 2011-HT-HGD-06.

\section{References}

1. Sun H., (2005) Research on Image Enhancement and Edge Detection Based on Mathematical Morphology, M.S.

Thesis, Dalian University of Technology,

2. Cui Q., (2001) Image processing and analysis-mathematical morphology method and application, BeiJing, Science Press.

3. Tang C.Q., (1993) Lv Hongbo, Huang Zheng, Mathematical morphology method and its application, BeiJing, Science Press.

4. Gong W., Shi Q.Y., (2000) Cheng Minde, Theory and application of mathematical space morphology, BeiJing, Science Press.

5. He D.J., Geng N., Zhang Y.K., (2003) Digital Image Processing, Shanxi Province: Xidian University Press.

6. Fan Linan, Han Xiaowei, Zhang G.Y., (2007) Image Processing and Pattern Recognition, BeiJing, Science Press.

7. Yang S.B., Peng F.Y., (2002) "Application of morphological edge detectors in image corrupted by noise",

Computer Engineering and Applicaions, vol.38, pp. 91-92. 